\title{
Direct Estimation of Quartz Microbalance Sensitivity by a Straight Optical Procedure
}

\author{
G. Magna ${ }^{1}$, F. Mandoj', F. Caso ${ }^{3}$, A. Catini ${ }^{1}$, R. Paolesse ${ }^{2}$, C. Di Natale ${ }^{1}$ \\ 1 Dipartimento Ingegneria Elettronica, Università di Roma Tor Vergata, Italy \\ 2 Dipartimento Scienze e Tecnologie Chimica, Università di Roma Tor Vergata, Italy \\ 3 ENEA, Casaccia Research Center, Roma, Italy \\ Corresponding author: magna.gabriele@gmail.com
}

\begin{abstract}
:
Quartz Microbalances (QMBs) are largely used in chemical sensor applications since their high mass sensitivity, good fabrication reproducibility and relative low cost. QMB sensitivity is generally calculated using the Sauerbrey equation. However experimental data can deviate from this model in case of uneven film profile, thick layers or elastic materials. In this work, the mass-frequency relationship has been measured in case of thick and porous layers of porphyrins. After porphyrin deposition, the layer is dissolved in a known THF volume and mass is estimated by UV-Vis spectra and Lambert-Beer law. Results show that the measured frequency-mass relationship has a good linear behavior in the typical application range and sensitivity is 1.25 times lower than the Sauerbrey equation prediction.
\end{abstract}

Key words: QMB, porphyrins, Lambert-Beer law.

\section{Introduction}

Gas sensitive porphyrins coated quartz microbalances have been fruitfully used since decades [1]. The interpretation of the behaviour of these sensors has been based on the straight application of the Sauerbrey equation [2]. However, due to uneven coating profile, finiteness of the piezoelectric disk, and the acoustic rigidity of the coating the frequency-mass relationship may deviate from the Saurbrey equation [3].

This work illustrates a simple procedure to measure the relationship between mass and frequency taking advantage from the optical properties of porphyrins. In addition to the wellknown chemical sensing properties, porphyrins are excellent dyes with molar extinction coefficients up to the order of $10^{5} \mathrm{~cm}^{-1} \mathrm{M}^{-1}$ [4]. Thanks to this property, UV-Vis spectroscopy techniques are able to detect very low porphyrin concentration (below $\mu \mathrm{M}$ ). Mass can be straight calculated when the solution volume is known.

\section{Experimental}

$20 \mathrm{MHz}$ Quartz Microbalances, $9 \mathrm{~mm}$ crystal diameter, $4 \mathrm{~mm}$ electrode diameter were used. predicts QMBs were connected to an oscillator circuit, the frequencies of the oscillators outputs are measured taking advantage of a

temperature compensated reference quartz that allows for a frequency resolution of $0.1 \mathrm{~Hz}$.

Multiple deposition of Zn-TetraPhenylPorhyrins have been made onto the QMB surface by spray casting technique in tetrahydrofuran (THF) solution. The QMB coating was performed through a mask that limits the coating onto the central electrode region which is supposed to be the sensitive region of the QMB. As showed by Figure 2, the coating is not uniform due Marangoni effect during solvent evaporation and likely to surface roughness. For each deposition, after measuring the frequency shift due to the coating, the porphyrin layer was removed, washing the QMB with THF. Porphyrins dissolved in THF was collected in a cuvette until to reach $3 \mathrm{ml}$. Since the molar extinction coefficient of the porphyrin is known, the concentration in the solution can be straightly calculated from the Beer-Lambert law.

$\ln \left(\frac{I}{I_{0}}\right)=-\varepsilon_{\lambda} C l$

Where $A$ is the absorbance, $I(\lambda)$ and $I_{0}(\lambda)$ the spectral intensity of light after and before the sample, $c$ is the concentration, $L$ is the length of 
the cuvette and $\varepsilon_{\lambda}$ is the molar extinction coefficient.

Figure 3 shows the experimental and theoretical mass-frequency relationship. The measured sensitivity is $5.78 \mathrm{~Hz} / \mathrm{ng}$. This value is 1.25 times smaller than the theoretical sensitivity calculated from the Sauerbrey equation. Noteworthy, the linearity of the massfrequency relationship ensures that porphyrins coated QMBs operates in the mass sensing regime.

This simple method can be promptly used to characterize the mass sensitivity of devices, such as cantilevers. for which a straight Saurbrey equation is not simply available.

\section{References}

[1] R. Paolesse, S. Nardis, D. Monti, M. Stefanelli, C. Di Natale, Porphyrinoids for chemical sensors applications. Chem Rev 117, 2517-2583 (2017) doi: 10.1021/acs.chemrev.6b00361

[2] G. Sauerbrey, Verwendung von Schwingquarzen zur Wägung dünner Schichten und zur Mikrowägung, Z. Physik 155, 206-222 (1959) doi: 2481/10.1007/BF01337937

[3] R. Lucklum, C. Behling, P. Hauptmann, Role of mass accumulation and viscoelastic film properties for the response of acoustic-wavebased chemical sensors, Analytical Chemistry, 71(13), 2488-2496, (1999); DOI: 10.1021/ac981245I.

[4] V. M. Albers, H. V. Knorr, Spectroscopic studies of the simpler porphyrins. J. Chem. Phys. 4, 422425, (1936); DOI: 10.1063/1.1723651

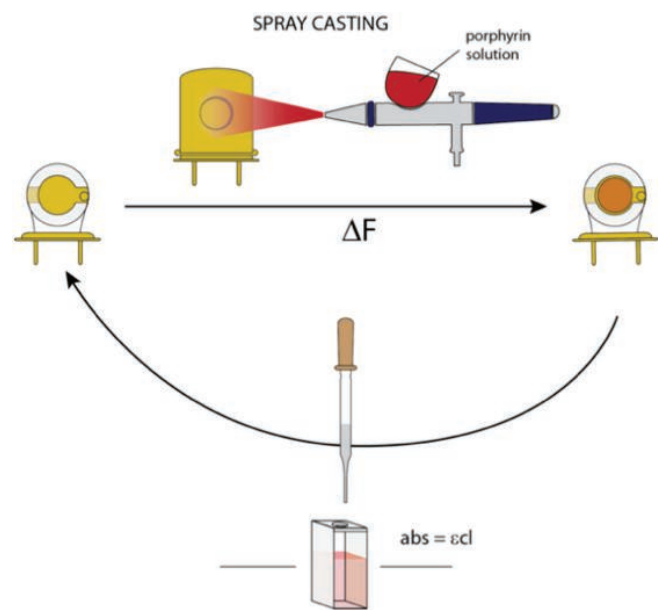

Fig. 1. Pictorial sketch of the method used to estimate the deposited mass. The coating, after deposition onto the $Q M B$, is dissolved back in its solvent (THF). The total mass is estimated from the Beer-Lambert equation.

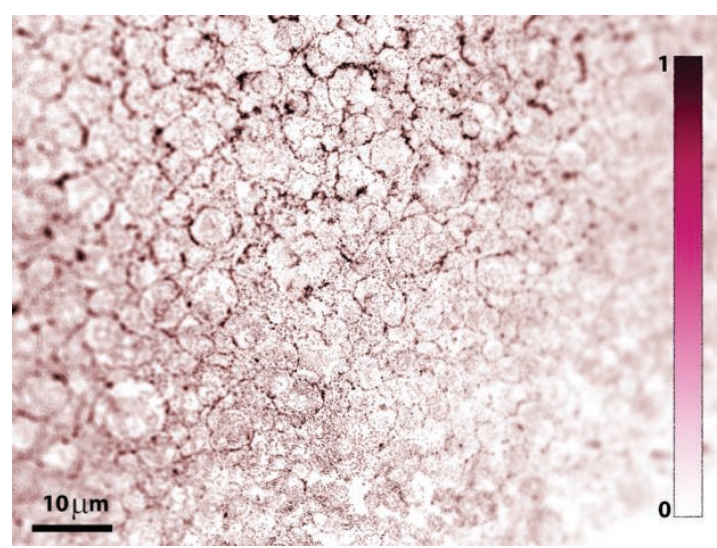

Fig. 2. Fluorescence microscopy of a portion of the porphyrin surface $(90 \mu \mathrm{m})$ shows a granular structure of the film. The shape can be attributed to both the Marangoni effect on each single droplet of spray casting and likely to the underlying granularity of the surface of the gold electrode.

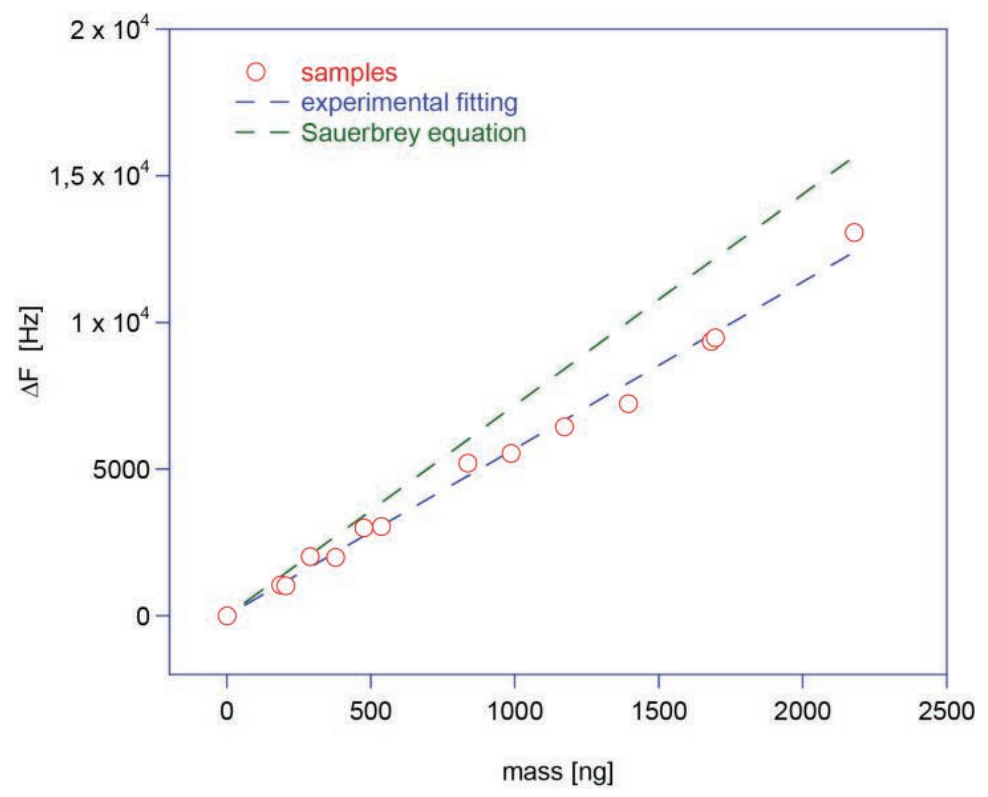

Fig.3. The experimentally measued masses linearly fits with the frequency shift. The experimental sensitivity is $5.78 \mathrm{~Hz} / \mathrm{ng}$ just 1.25 times smaller than the theoretic value calculated applying the Sauerbrey equation and assuming that the area of the electrode is the effectively sensitive region of the QMB. 